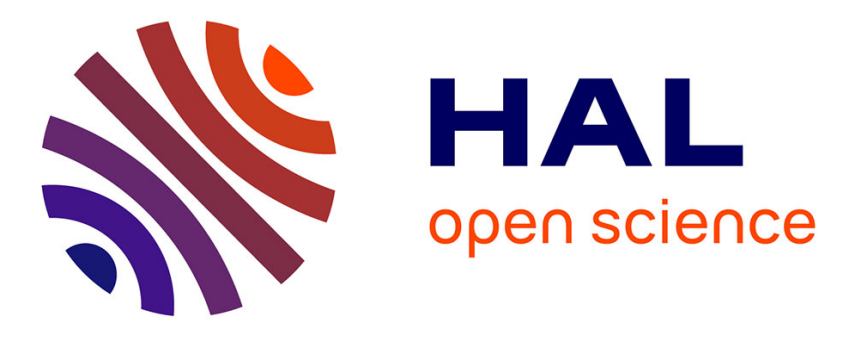

\title{
The case for seasonal surface changes at Titan's lake district
}

Shannon Mackenzie, Jason Barnes, Jason Hofgartner, Samuel Birch, Matthew

Hedman, Antoine Lucas, Sébastien Rodriguez, Elizabeth Turtle, Christophe

Sotin

\section{To cite this version:}

Shannon Mackenzie, Jason Barnes, Jason Hofgartner, Samuel Birch, Matthew Hedman, et al.. The case for seasonal surface changes at Titan's lake district. Nature Astronomy, 2019, 3 (6), pp.506-510. 10.1038/s41550-018-0687-6 . hal-02330630

\section{HAL Id: hal-02330630 \\ https://hal.science/hal-02330630}

Submitted on 13 Nov 2020

HAL is a multi-disciplinary open access archive for the deposit and dissemination of scientific research documents, whether they are published or not. The documents may come from teaching and research institutions in France or abroad, or from public or private research centers.
L'archive ouverte pluridisciplinaire HAL, est destinée au dépôt et à la diffusion de documents scientifiques de niveau recherche, publiés ou non, émanant des établissements d'enseignement et de recherche français ou étrangers, des laboratoires publics ou privés. 


\title{
The case for seasonal surface changes at Titan's lake district
}

\author{
Shannon M. MacKenzie*1,2, Jason W. Barnes², Jason D. Hofgartner ${ }^{3}$, \\ Samuel P.D. Birch ${ }^{4}$, Matthew M. Hedman ${ }^{2}$, Antoine Lucas ${ }^{5}$, \\ Sebastien Rodriguez ${ }^{6}$, Elizabeth P. Turtle ${ }^{1}$, Christophe Sotin $^{3}$
}

December 14, 2018

\begin{abstract}
1 Applied Physics Laboratory, John Hopkins University, Laurel, MD 20723, United States ${ }^{2}$ Department of Physics, University of Idaho, Moscow, ID 83844, United States ${ }^{3}$ Jet Propulsion Laboratory/California Institute of Technology, Pasadena, CA 91109, United States ${ }^{4}$ Department of Astronomy, Cornell University, Ithaca, NY 14850, United States ${ }^{5}$ AIM CEA-Saclay, Universit Paris Diderot, USPC, Paris, France ${ }^{6}$ Institut de Physique du Globe de Paris (IPGP) Universit Paris Diderot, Paris, France
\end{abstract}


Titan, Saturn's largest moon, hosts lakes and seas of liquid hydrocarbons at its poles ${ }^{1}$. General circulation models demonstrate that regional evaporation and precipitation rates of methane likely change with the seasons (Titan's year $\sim 29.5$ Earth years) and evolve on a geological timescale $\left(\sim 10^{5} \text { Earth years }\right)^{2 / 34}$. Cassini observations suggest shoreline recession at a few south polar lakes during local summer ${ }^{3}$, but similar seasonal changes have yet to be observed at the north pole where lakes are larger and more numerous $6 / 3 / 7$. We present three "phantom lakes" that appear to be north polar surface liquids in winter observations by Cassini RADAR but which are inconsistent with lakes in infrared images obtained up to 7 years later, after vernal equinox, suggesting that the liquids were removed in between. If this were the case, the phantom lakes could be interpreted as shallow ponds, with either a pure methane composition or a regolith porous enough to remove the less volatile ethane. These phantom lakes provide observational constraints on removal timescales for surface liquids at Titan's north pole. The location, size, and longevity of surface liquid reservoirs affect sediment processing ${ }^{7}$, seasonal weather ${ }^{\mathbf{8}}$, climate evolution ${ }^{\mathbf{9}}$, and even, perhaps, their habitability 10 . As solubility of the possible non-polar mixtures is generally low, short-lived lakes might be nutrient-poor ${ }^{10}$ and thus have low astrobiological potential.

Complete emptying of small or shallow lakes may be the easiest shoreline change to identify in Cassini data, given that the largest predicted evaporation and infiltration rates peak at meters per year $11 / 6 \mid 12$. For example, though observations of Ontario Lacus, Titan's largest south polar lake, were interpreted as evidence of kilometers of shoreline recession over four years 8 , later analysis ${ }^{14}$ contested that such change was within the uncertainty of spatial sampling. The difficulty in identifying even the most dramatic liquid level change lies in establishing a long enough baseline between observations with sufficient spatial resolution to discern changes. Data from a single instrument are rarely able to produce suitable comparisons for this purpose. But, by combining data from RADAR, the Visual and Infrared Mapping Spectrometer (VIMS) and the Imaging Science Subystem (ISS) we can take advantage of 13 years of Titan-targeted flybys at the cost of added complexity.

Each instrument's dataset is unique in spatial and temporal resolution and coverage, making precise comparison between them nontrivial. Furthermore, the instruments probe different properties of the surface. VIMS and ISS are sensitive to composition and grain size on the scale of a few microns while Synthetic Aperture RADAR (SAR) images convey information about dielectric properties and surface roughness on the centimeter scale.

Lakes are identifiable due to their lacustrine morphology and characteristic darkness in each dataset. Light specularly reflects off the surface of the smooth lakes ${ }^{1521}$, often away from the direction of Cassini. In the infrared (IR), diffuse scattering off haze in the atmosphere also provides some illumination, making lakes appear dark grey to ISS $(0.93 \mu \mathrm{m})$ and dark blue to VIMS $(0.9$ $\mu \mathrm{m}-5 \mu \mathrm{m})$ in the color scheme of Figures 1 and 2 . At $2.17 \mathrm{~cm}$, the observed RADAR signal is unaffected by the atmosphere ${ }^{17}$ and is a combination of quasispecular backscatter and diffuse backscatter ${ }^{15}$. For lakes, SAR observes low signal return resulting from little to no quasispecular contribution (due to the smooth lake surface); weak diffuse contribution from volume scattering within the liquid if it is nearly homogenous liquid; and reduced scattering off the lake floor boundary (due to the small Fresnel reflection coefficient between liquid and solid organics) 18 . Attenuation within a liquid column begins to affect the signal when path lengths are greater than tens of meters $^{19 \mid 20}$ (Supplemental Figure 1). Each instrument is sensitive to liquid depths of at least a few wavelengths.

The location and extent of Titan's lakes and seas are thus some of the strongest cases of agreement between RADAR, VIMS, and ISS datasets. Three surface features, however, defy that 
agreement. These "phantom lakes" appear liquid-filled and lacustrine in shape to RADAR but not to the IR instruments in later observations. Observed by RADAR in SAR mode on July 22 and October 9 of $2006^{1}$ (top panel, Figure 2), the phantom lakes are dark in both the denoised ${ }^{2}$ images (Figure 1) and raw images (Figure 2). Their normalized radar cross sections $\left(\sigma^{0}\right)$ are more consistent with nearby lakes than dry surfaces. In Figure 3 we show the $\sigma^{0}$ of the phantom lakes relative to empirical fits of empty lakes (red) and partially-filled lakes (blue); shading represents $95 \%$

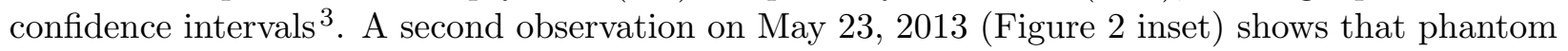
lake $B$ remained dark enough to agree with the lake fit, though is brighter than predicted.

Of course, RADAR darkness does not a lake make. We do not definitively rule out that some combination of smoothness or reduced volumetric scattering ${ }^{22}$ makes these features RADAR-dark, dry surfaces. However, the lacustrine morphology of each candidate makes the presence of liquids a more likely explanation, especially given the phantom lakes' locations within the lake district and far from, for example, the dark dunes of the equatorial region. While phantom lake A lacks the sharp edges seen at some lakes ${ }^{23}$, it is locally confined at a scale similar to neighboring lakes and has diffuse boundaries like those seen at Jingpo and MacKay Lacus; A is therefore unlikely to be a dark plain (examples of which are plotted as grey circles in Figure 3).

Phantom lake B is a dark area along the southern shoreline of Oneida Lacus, a lake confirmed to have liquids by echoes in the April 2017 altimetry pass (which unfortunately ends before reaching phantom lake B). Overlap between SAR swaths T19 and T91 indicates that the rim extends partially along the southern shore, separating half of the phantom lake terrain from the liquid of Oneida. Thus, phantom lake B's morphology suggests a plain in contact with a lake shoreline, potentially a flood plain. Finally, C's boundaries resemble those of neighboring sharp-edge depressions ${ }^{7}$.

Because the ISS and VIMS cameras could not observe Titan's north pole until the Sun rose with northern spring (August 2009) and the winter clouds dissipated 24 . ISS first glimpsed the lake district on October 13, 2013. In that image, phantom lake A was not dark like a lake while phantom lakes $\mathrm{B}$ and $\mathrm{C}$ were not resolved. A month later (Figure 2 middle), phantom lakes $\mathrm{A}$ and $\mathrm{C}$ appear bright, inconsistent with the presence of surface liquids. The observation of phantom lake B is less conclusive due to its proximity to Oneida Lacus.

VIMS followed up on December 1, 2013 (Figure 2 bottom). At as fine as $4 \mathrm{~km} /$ pixel, at least four spectral units-surface materials with similar reflectance properties in the IR-are evident. Evaporites $^{25 \mid 26}$ appear bright pink/orange in our infrared color images because of their diagnostic brightness at $5 \mu \mathrm{m}$; they surround some of the dark blue lakes. (The blue and green materials are spectrally distinct but not yet geomorphologically interpreted.) VIMS pixels covering phantom lakes A and B do not behave as a single unit as they do in RADAR.

In Figure 4 we show that the southern pixels of A (black square) are darker than the northern pixels (grey square). Similarly, the eastern half of B (grey star) is darker than the western (black star). These bright western pixels of B parallel the raised rim of Oneida Lacus. Though the outline of the phantom lakes is unmistakeable in RADAR, A and $\mathrm{C}$ do not show these boundaries in VIMS. Phantom lake B's two VIMS units do correspond to the RADAR boundaries, providing morphological evidence that this RADAR-dark patch is a unique and isolated feature. Phantom lake C's spectrum most closely resembles the evaporites of Ypoa and Suwa Lacus, nearby lakes that appear liquid filled in all datasets.

Subsequent VIMS observations are too coarse for in-depth analysis but appear consistent with a lack of surface liquids. ISS images from February 2014 and July 2017 show that the phantom lakes are bright even in the final Cassini glimpses of the lake district.

Thus, the phantom lakes scatter like nearby solid surfaces in the infrared. They cannot be open bodies of liquid at the time of VIMS or ISS observations. How then can we reconcile the liquid radar-darkness with an IR bright surface? We put forth three possible explanations for the 
phantom lake observations: (1) constant liquid level with a RADAR-invisible topmost layer, (2) an IR scattering layer developing between observations, and (3) liquid removal from at least the top few microns of the surface. In all cases, the radar-darkness arises from a liquids on or saturating the surface at the time of the RADAR observations.

In scenario (1), no surface change occurs. Rather, the difference between the RADAR and IR observations stems from the difference in wavelengths observed by the RADAR mapper and the IR imagers. For example, the diffuse scattering off suspended Rayleigh particles much smaller than a RADAR wavelength with dielectric constants near that of liquid methane could contribute a small enough $\sigma^{0}$ that the lake would appear radar-dark ${ }^{18}$. In this case, the scattering layer must be less than a RADAR wavelength $(2.17 \mathrm{~cm})$ to be effectively transparent to the instrument.

In scenario (2), the scattering layer could develop in the time between the RADAR and IR observations. In this case, the IR bright layer need only be thick enough to obscure the the liquid below.

The scattering "scum" layer could be bubbles, haze fallout, or suspended sediment atop a liquid layer. But, as even a few mm of liquid would show significantly decreased reflectance to VIMS ${ }^{27}$, the overall IR brightness of the phantom lakes requires that the scattering layer be optically thick (a few $\mathrm{mm}$ ). Furthermore, as no ethane absorption features are present in any of the phantom lakes' spectra, ethane cannot be present in the scattering layer (Supplemental Figure 3). This, along with the fact that the phantom lakes do not exhibit the same boundaries in VIMS as they do in RADAR, suggests that bubbles, haze fallout, and suspended sediment are unlikely explanations for the IR brightness of the phantom lakes.

Alternatively, a dry rind sitting atop a saturated regolith would not necessarily show unique spectral boundaries and is thus the more likely no-change scenario. Huygens observations ${ }^{[28}$ suggest even Titan's equatorial desert has liquid methane close to the surface. Modeling Titan's climate with extensive surface liquid reservoirs better reproduces the observed distribution of seas and cloud activity $2 \mid 12 / 429$. The phantom lakes could therefore be highly localized areas of near surface wetted regolith distinct from the surrounding features, an explanation perhaps more likely for phantom lake B given its morphological similarity to a flood plain of Oneida Lacus and less likely for A and C given their lacustrine morphologies and distance from other lakes. If the phantom lakes are the first Cassini identification of regolith saturated up to the top few $\mathrm{mm}$, it is curious that such saturated regolith is limited to a few isolated locales given that lakes are widely distributed throughout the polar region.

We therefore currently favor (3) in which liquids are responsible for the dark $\sigma^{0}$ and are removed to at least a few mm below the surface by the time VIMS and ISS observe the phantom lakes. A freshly exposed solid surface could explain both the IR brightness and the similarity between the phantom lakes' VIMS units and those of the surrounding terrain. Processes for emptying a lake on Titan include draining by channels, infiltration into a porous regolith, and evaporation. Cassini could not resolve any channels feeding into the phantom lakes; if any such features exist they must be subpixel in size $(<300 \mathrm{~m})$.

For features $\mathrm{A}$ and $\mathrm{C}$, the emptying process would have to take place within the seven years between observations, a timescale that easily fits within the estimates for emptying via a combination of evaporation and infiltration. If the phantom lakes are shallow, evaporation would empty them more quickly than a nearby deep lake-creating more easily resolvable shoreline change-and should pick up with increased insolation during northern summer, in line with the sequence of our observations. Infiltration can happen on relatively short timescales: even the least permeable regoliths hypothesized on Titan can drain lakes of $10 \mathrm{~m}$ to a methane table a few centimeters below the surface within several years ${ }^{3}$. Modeled evaporation rates for Titan range from $\sim 0.01 \mathrm{~mm} /$ Earth year $\underline{12}$ to meters/Earth year $\underline{11}$. The most conservative evaporation estimates $(0.01 \mathrm{~mm} /$ Earth year $)$ 
constrain the evaporated volumes of phantom lakes $\mathrm{A}$ and $\mathrm{C}$ to as small as $10^{6} \mathrm{~m}^{3}$; corresponding to depths too shallow to be sufficiently dark to RADAR. Thus, infiltration of about the same volume would have to have played a role.

Feature B, on the other hand, would have a much shorter timescale for change. Though brief, the six months between observations may be enough time to remove liquid from the shallow depths of the surface that the IR instruments sense. Phantom lake B appears brighter to RADAR at higher incidence angles than would be expected for a shallow lake. Scattering off suspended particles can increase $\sigma^{0}$, but so can looking through less liquid, i.e. a decrease in the amount of liquid at phantom lake B. Furthermore, B is situated along the shoreline of Oneida Lacus, enhancing the likelihood of a shallow deposition of liquid (e.g. lake overflow) or draining into a nearby reservoir (e.g. deeper into the subsurface). As a raised rim separates the western half of phantom lake B from Oneida Lacus, it is more likely for liquid to overflow on the eastern half, consistent with the difference in VIMS units.

Together, the phantom lakes suggest that observable change may have occurred at Titan's north pole during the Cassini mission at timescales (6 months - 7 years) comparable to that observed for south polar lakes ${ }^{3}$ of similar size. This seasonal timescale would also be consistent with the range of removal rates predicted for evaporation ${ }^{11 / 12}$ and infiltration ${ }^{6}$ when operating in conjunction. Barring substantial local (less than a few $\mathrm{km}$ ) differences in evaporation and/or infiltration properties, we expect that larger and deeper lakes are exchanging their liquids between the surface and atmosphere at similar rates, but their greater depths make their shoreline charges undetectable by Cassini.

\section{References}

[1] Stofan, E. R. et al. The lakes of Titan. Nature 445, 61-64 (2007).

[2] Lunine, J. I. \& Lorenz, R. D. Rivers, lakes, dunes, and rain: crustal processes in Titan's methane cycle. Annu. Rev. Earth Planet. Sci 37, 299-320 (2009).

[3] Lora, J. M., Lunine, J. I., Russell, J. L. \& Hayes, A. G. Simulations of Titan's paleoclimate. Icarus 243, 264-273 (2014).

[4] Mitchell, J. L. \& Lora, J. M. The Climate of Titan. Annu. Rev. Earth Planet. Sci 44, 353-380 (2016).

[5] Hayes, A. G. et al. Transient surface liquid in Titan's polar regions from Cassini. Icarus 211, 655-671 (2011).

[6] Hayes, A. et al. Hydrocarbon lakes on Titan: Distribution and interaction with a porous regolith. Geophys. Res. Lett. 35, L9204 (2008).

[7] Birch, . D., S. P et al. Geomorphologic mapping of titan's polar terrains: Constraining surface processes and landscape evolution. Icarus 282, 214-236 (2017).

[8] Tokano, T. Impact of seas/lakes on polar meteorology of Titan: Simulation by a coupled GCM-Sea model. Icarus 204, 619-636 (2009).

[9] Lora, J. M., Lunine, J. I. \& Russell, J. L. GCM simulations of Titan's middle and lower atmosphere and comparison to observations. Icarus 250, 516-528 (2015). 
[10] Tokano, T. Limnological structure of Titan's hydrocarbon lakes and its astrobiological implication. Astrobiology 9, 147-164 (2009).

[11] Mitri, G., Showman, A. P., Lunine, J. I. \& Lorenz, R. D. Hydrocarbon lakes on Titan. Icarus 186, 385-394 (2007).

[12] Newman, C. E., Richardson, M. I., Lian, Y. \& Lee, C. Simulating Titan's methane cycle with the TitanWRF general circulation model. Icarus 267, 106-134 (2016).

[13] Turtle, E. P., Perry, J. E., Hayes, A. G. \& McEwen, A. S. Shoreline retreat at Titan's Ontario Lacus and Arrakis Planitia from Cassini Imaging Science Subsystem observations. Icarus 212, 957-959 (2011).

[14] Cornet, T. et al. Edge detection applied to Cassini images reveals no measurable displacement of Ontario Lacus' margin between 2005 and 2010. J. Geophys. Res. E: Planets 117, 7005 (2012).

[15] Wye, L. C. et al. Electrical properties of Titan's surface from Cassini RADAR scatterometer measurements. Icarus 188, 367-385 (2007).

[16] Soderblom, J. M. et al. Modeling specular reflections from hydrocarbon lakes on Titan. Icarus 220, 744-751 (2012).

[17] Rodriguez, S. et al. Impact of aerosols present in Titan's atmosphere on the Cassini radar experiment. Icarus 164, 213-227 (2003).

[18] Hofgartner, J. D. et al. Titan's "Magic Islands": Transient features in a hydrocarbon sea. Icarus 271, 338-349 (2016).

[19] Mastrogiuseppe, M. et al. The bathymetry of a Titan sea. Geophys. Res. Lett. 41, 1432-1437 (2014).

[20] Mitchell, K. L., Barmatz, M. B., Jamieson, C. S., Lorenz, R. D. \& Lunine, J. I. Laboratory measurements of cryogenic liquid alkane microwave absorptivity and implications for the composition of ligeia mare, titan. Geophysical Research Letters 42, 1340-1345 (2015).

[21] Lucas, A. et al. Insights into Titan's geology and hydrology based on enhanced image processing of Cassini RADAR data. J. Geophys. Res. E: Planets 119, 2149-2166 (2014).

[22] Paillou, P., Crapeau, M., Elachi, C., Wall, S. \& Encrenaz, P. Models of synthetic aperture radar backscattering for bright flows and dark spots on Titan. J. Geophys. Res. E: Planets 111, E11 (2006).

[23] Birch, S. et al. Raised rims around titan's sharp-edged depressions. Geophysical Research Letters (2018).

[24] Le Mouélic, S. et al. Dissipation of Titan's north polar cloud at northern spring equinox. Planet. Space Sci. 60, 86-92 (2012).

[25] Barnes, J. W. et al. Organic sedimentary deposits in Titan's dry lakebeds: Probable evaporite. Icarus 216, 136-140 (2011).

[26] MacKenzie, S. M. et al. Evidence of Titan's climate history from evaporite distribution. Icarus 243, 191-207 (2014). 
[27] Clark, R. N. et al. Detection and mapping of hydrocarbon deposits on Titan. J. Geophys. Res. E: Planets 115, 10005 (2010).

[28] Lorenz, R. D., Niemann, H. B., Harpold, D. N., Way, S. H. \& Zarnecki, J. C. Titan's damp ground: Constraints on Titan surface thermal properties from the temperature evolution of the Huygens GCMS inlet. Meteorit. Planet. Sci 41, 1705-1714 (2006).

[29] Turtle, E. P. et al. Titan's meteorology over the Cassini mission: Evidence for extensive subsurface methane reservoirs. Geophys. Res. Lett. (2018).

[30] Hayes, A. G. The lakes and seas of Titan. Annu. Rev. Earth Planet. Sci 44, 57-83 (2016).

\section{Acknowledgements}

This work was supported under the NASA Earth and Space Science Fellowship Program grant NNX14AO30H to SMM. JWB acknowledges support from NASA Cassini Data Analysis Program NNX15AI77G. AL and SR acknowledges the financial support of the UnivEarthS Labex program at Sorbonne Paris Cité (ANR-10-LABX-0023 and ANR-11-IDEX-0005-02). SR is also supported by the French National Research Agency (ANR-APOSTIC-11-BS56-002, ANR-12-BS05-001-3/EXODUNES). Part of this work was carried out at the Jet Propulsion Laboratory, California Institute of Technology, under contract with NASA. Copyright 2018. Government sponsorship is acknowledged.

\section{Author Contributions}

SMM led the procuring and analysis of the data and wrote the manuscript. JWB assisted in all aspects of the analysis and writing. JH and SPDB contributed to the analysis of RADAR data. MMH helped develop scattering models. AL wrote the code to produce NLDSAR and provided these data. All authors contributed to the discussion of these results. SR provided the radiative transfer model and contributed to their analysis. EPT assisted in the calibration of ISS data and planned the ISS observations. CS planned the VIMS Titan observations.

\section{Competing Interest}

The authors declare no competing interest.

*Correspondence or request for materials should be directed to SMM (shannon.mackenzie@jhuapl.edu).

\section{Figure legends}

Figure 1: Cassini images of Titan's north pole. (a) Azimuthal stereographic projection of Titan's north pole as observed by Cassini VIMS on December 13, 2013. Titan's hydrocarbon seas (maria) lie opposite the lake district and at lower elevation 3017 . (b-e) Eastern edge of the lake district as seen by VIMS (up to $4 \mathrm{~km} /$ pixel; b), ISS (map scale at $4 \mathrm{~km} /$ pixel; c), and Non-Localized Denoised ${ }^{2}$ SAR $(350 \mathrm{~m} /$ pixel; d). These instruments observed the eastern half of the lake district at different wavelengths and seasons (northern winter and northern spring). A,B, and C labels indicate the phantom lake features on the NLDSAR image. Relevant lake names are given on the ISS map.

Figure 2: Sequence of observations of the phantom lakes, outlined in each dataset. SAR coverage from T16 and T19 (a) and T91 (b) show the phantom lakes as dark. But later, the same areas 
appear bright in ISS (c) and VIMS (d) images. Normally, lakes appear dark in all three datasets. Because our temporal coverage relies on three datasets that probe different surface properties, we consider both liquid level change and physical phenomena as possible explanations.

Figure 3: Normalized radar cross sections $\left(\sigma^{0}\right)$ from SAR swaths as a function of incidence angle for the phantom lakes $(A, B, C)$; lakes (blue circles); empirical fits for partially-filled lakes (blue) and empty lakes (red) with $95 \%$ confidence intervals shaded ${ }^{3}$; and non-lacustrine, uniform, SAR-dark plains $^{7}$ (dark grey circles). $O$ and $U$ represent the $\sigma^{0}$ of Oneida Lacus and an unnamed adjacent lake, respectively; each have altimetry returns consistent with a specular reflection off a smooth, presumably liquid surface. $\sigma^{0}$ is a measure of the microwave reflectivity of a material. The standard errors for each feature are smaller than the plotted symbols. The flyby number for each of the two $\mathrm{B}$ and $\mathrm{U}$ observations are indicated in subscripts.

Figure 4: Behavior of phantom lakes relative to other VIMS spectral units within the atmospheric windows. Because VIMS can only see down to Titan's surface in seven windows $(0.93,1.0,1.3$, $1.6,2.0,2.7,2.8$, and $5 \mu \mathrm{m}$ ), we group surface materials into spectral units defined by the relative reflectance at these wavelengths (b,c). In the T96 VIMS image (CM_1764550739_16; a), we identify four units (lake in purple, evaporite in pink, green, and blue) as endmembers to which we compare phantom lake pixels (examples labeled in top image). Phantom lakes A and B have two groups of pixels (black, and grey), suggesting two spectral units within the area that RADAR sees as a dark unit. Atmospheric effects have been mitigated with a radiative transfer model. 


\section{Methods}

After calibration (described below), we imported images into QGIS, an open-source GIS, for projection and mapping.

In Synthetic Aperture Radar (SAR) mode, the Cassini RADAR instrument mapped the surface at resolutions of $\sim 350 \mathrm{~m} /$ pixel at $2.17 \mathrm{~cm}^{1}$. We use incidence angle, noise-subtracted, and $\mathrm{NLDSAR}^{2]}$ swaths from T16, T19, and T91. Incidence and $\sigma^{0}$ values were extracted via polygon mapping and statistical tools within QGIS. The fits shown in Figure 3 are taken directly from a previously published empirical fit to SAR data ${ }^{3}$.

ISS imaged Titan at $0.938 \mu \mathrm{m}^{4}$. At this wavelength, haze particles in the atmosphere scatter most of the light before it reaches the surface. However, the shape of the haze phase scattering function ${ }^{5}$ actually facilitates observation of the surface because some of that light forward scatters to the ISS detector. The resulting ISS maps are generally $1 \mathrm{~km}$ per pixel. Thus, ISS data have been used to identify the presence of liquid on Titan's surface $e^{6 / 78}$ as well as monitor cloud activity in the atmosphere ${ }^{6 / 9}$. For this work, we used the global mosaic map available from the USGS, as well as data from October 13, 2013 to February 2, 2014 available on the PDS. The latter were calibrated and mosaicked using USGS Integrated Software for Imagers and Spectrometers (ISIS).

VIMS observed Titan in the near infrared windows at which methane absorption (and that of trace gases) is weak enough to allow light to penetrate through the atmosphere and interact with the surface 10111: $0.93 \mu \mathrm{m}, 1.0 \mu \mathrm{m}, 1.3 \mu \mathrm{m}, 1.6 \mu \mathrm{m}, 2.0 \mu \mathrm{m}, 2.7 \mu \mathrm{m}, 2.8 \mu \mathrm{m}$, and $5 \mu \mathrm{m}$. Though specific chemical composition is difficult to identify 12113 , the diverse surface can be divided by spectral reflectance properties into spectral units 14 .

VIMS data were calibrated and colored $\frac{14}{14}$ for initial identification of spectral units, shown in Figures 1 and 2. We identified four spectral units in the high resolution cube-lake, evaporite, blue, and green-based on the unique behavior of the band ratios. These endmember representative spectra were created by averaging over pixels within the identified units. To rule out atmospheric phenomena, we applied an empirical correction ${ }^{15}$ and also modeled the spectra with the radiative transfer model $16|17| 18$. In both data sets, the spectral behavior of the phantom lakes shown in Figure 4 is consistent and agrees with the raw data. The radiative transfer model, however, allowed us to significantly reduce the standard deviation of the spectral ratios with respect to the raw and empirically corrected data, thus reinforcing the conclusions that the phantom lakes are not liquid filled in the VIMS data.

The radiative transfer model used is that of Hirtzig et al. $(2013)^{\underline{16}}$ with a look-up-table scheme $e^{17}$ as described in detail by Brossier et al. (2018) 18 . Though the model relies on Huygens ground truth from the equatorial region, the decreased standard deviation of the spectral ratios of all units indicates that the radiative transfer model fairly mitigated the atmospheric contributions, one of the major sources of data variability.

There are many IR bright phenomena in VIMS observations of Titan. Specular reflections off

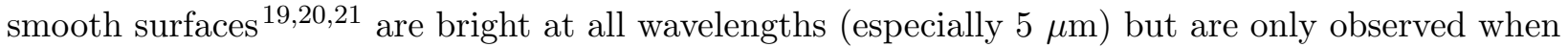
Cassini images the surface at the same angle at which the light is incident. None of our phantom lakes were imaged at this geometry. Broad specular reflections from wavy surfaces can occur further from the specular point but are also much brighter than the reflectance of the phantom lakes ${ }^{22}$. Furthermore, no specular reflections have been identified in ISS data to date, as the the high optical depth at $0.93 \mu \mathrm{m}$ makes direct surface scattering unlikely even at favorable geometries. Thus, neither direct nor broad specular reflections can explain the brightness of the phantom lake features to ISS. Clouds are also bright but have a distinct, ephemeral spectral signature $23|24| 25 \mid 26$ that is inconsistent with that of the phantom lakes (Supplemental Figure 6). As the phantom lakes do not appear to drastically change between observations (October 2013- July 2017) with 
VIMS and ISS, clouds are unlikely to hide liquids. The adjacency effect can artificially brighten dark surfaces when photons reflected from nearby bright surfaces get scattered into the cone of acceptance of the pixel imaging the dark material. However, this effect dominates at shorter distances with increasing optical depth $\frac{27}{27}$ and we observe the opposite: the phantom lakes appear brighter at the longer wavelengths. The brightness of the phantom lakes is therefore most likely due to compositional and grain size properties of the surface and not some observational or atmospheric phenomenon.

\section{Data Availability}

All data presented here are available from the NASA Planetary Data System

(https://pds-imaging.jpl.nasa.gov/portal/cassini_mission.html), except the NLDSAR swaths which are maintained by coauthor Lucas (http://cssnldsar.geophysx.org/). The data that support the plots within this paper are also available from the corresponding author upon reasonable request.

\section{References}

[1] Elachi, C. et al. Radar: the Cassini Titan radar mapper. Space Sci. Rev. 115, 71-110 (2004).

[2] Lucas, A. et al. Insights into Titan's geology and hydrology based on enhanced image processing of Cassini RADAR data. J. Geophys. Res. E: Planets 119, 2149-2166 (2014).

[3] Hayes, A. G. et al. Transient surface liquid in Titan's polar regions from Cassini. Icarus 211, 655-671 (2011).

[4] Porco, C. C. et al. Cassini imaging science: instrument characteristics and anticipated scientific investigations at Saturn. Space Sci. Rev. 115, 363-497 (2004).

[5] Tomasko, M. G. et al. A model of Titan's aerosols based on measurements made inside the atmosphere. Planet. Space Sci. 56, 669-707 (2008).

[6] Turtle, E. P. et al. Cassini imaging of Titan's high-latitude lakes, clouds, and south-polar surface changes. Geophys. Res. Lett. 36, L2204 (2009).

[7] Turtle, E. P. et al. Rapid and extensive surface changes near Titan's equator: evidence of April showers. Science 331, 1414-1417 (2011).

[8] Turtle, E. P., Perry, J. E., Hayes, A. G. \& McEwen, A. S. Shoreline retreat at Titan's Ontario Lacus and Arrakis Planitia from Cassini Imaging Science Subsystem observations. Icarus 212, 957-959 (2011).

[9] Turtle, E. P. et al. Seasonal changes in Titan's meteorology. Geophys. Res. Lett. 38, 3203 (2011).

[10] Baines, K. H. et al. The atmospheres of Saturn and Titan in the near-infrared: First results of Cassini/VIMS. Earth Moon and Planets 96, 119-147 (2005).

[11] Brown, R. H. et al. Observations in the Saturn system during approach and orbital insertion, with Cassini's visual and infrared mapping spectrometer (VIMS). A \& A 446, 707-716 (2006). 
[12] McCord, T. B. et al. Titan's surface: Search for spectral diversity and composition using the Cassini VIMS investigation. Icarus 194, 212-242 (2008).

[13] MacKenzie, S. M. \& Barnes, J. W. Compositional similarities and distinctions between Titan's evaporitic terrains. Aptrophys. J. 821, 17 (2016).

[14] Barnes, J. W. et al. Global-scale surface spectral variations on Titan seen from Cassini/VIMS. Icarus 186, 242-258 (2007).

[15] Le Mouélic, S. et al. Global mapping of Titan's surface using an empirical processing method for the atmospheric and photometric correction of Cassini/VIMS images. Planet. Space Sci. 73, 178-190 (2012).

[16] Hirtzig, M. et al. Titan's surface and atmosphere from Cassini/VIMS data with updated methane opacity. Icarus 226, 470-486 (2013).

[17] Maltagliati, L. et al. Titan's atmosphere as observed by Cassini/VIMS solar occultations: $\mathrm{CH}_{4}, \mathrm{CO}$ and evidence for $\mathrm{C}_{2} \mathrm{H}_{6}$ absorption. Icarus 248, 1-24 (2015).

[18] Brossier, J. F. et al. Geological evolution of Titan's equatorial regions: possible nature and origin of the dune material. J. Geophys. Res. E: Planets (2018).

[19] Stephan, K. et al. Specular reflection on Titan: liquids in Kraken Mare. Geophys. Res. Lett. 37 (2010).

[20] Barnes, J. W. et al. Wave constraints for Titan's Jingpo Lacus and Kraken Mare from VIMS specular reflection lightcurves. Icarus 211, 722-731 (2011).

[21] Soderblom, J. M. et al. Modeling specular reflections from hydrocarbon lakes on Titan. Icarus 220, 744-751 (2012).

[22] Barnes, J. W. et al. Cassini/VIMS observes rough surfaces on Titan's Punga Mare in specular reflection. Planetary science 3, 3 (2014).

[23] Griffith, C. A. et al. The evolution of Titan's mid-latitude clouds. Science 310, 474-477 (2005).

[24] Barnes, J. W. et al. VIMS spectral mapping observations of Titan during the Cassini prime mission. Planetary and Space Science 57, 1950-1962 (2009).

[25] Rodriguez, S. et al. Global circulation as the main source of cloud activity on Titan. Nature 459, 678-682 (2009).

[26] Rodriguez, S. et al. Titan's cloud seasonal activity from winter to spring with Cassini/VIMS. Icarus 216, 89-110 (2011).

[27] Minomura, M., Kuze, H. \& Takeuchi, N. Adjacency effect in the atmospheric correction of satellite remote sensing data: Evaluation of the influence of aerosol extinction profiles. Opt. Rev. 8, 133-141 (2001). 



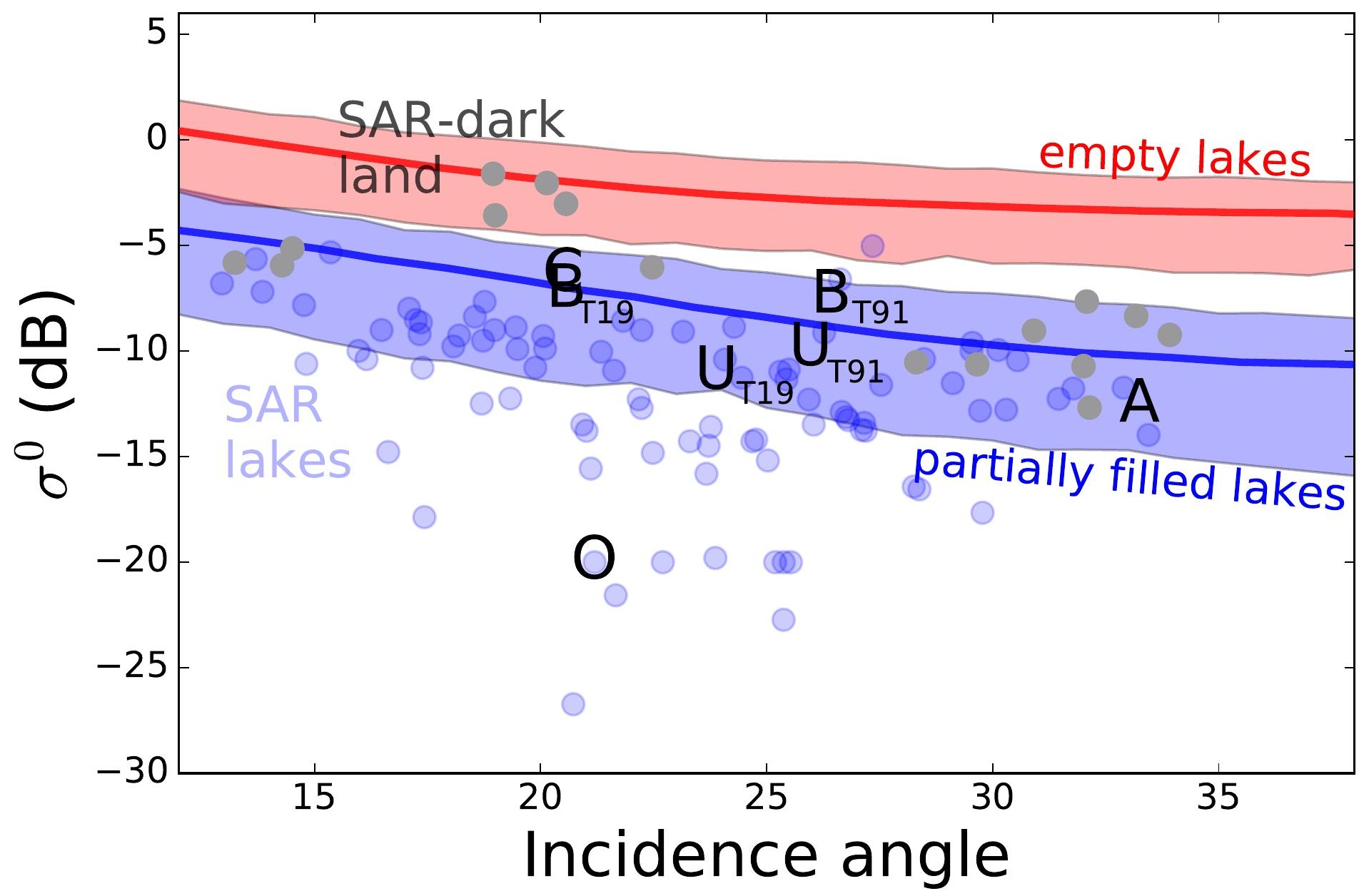


